\title{
NinA BEREND
}

\section{Regionale Gebrauchsstandards - Gibt es sie und wie kann man sie beschreiben?*}

\begin{abstract}
Der Beitrag beschäftigt sich mit der Frage, ob es im Deutschen regionale Gebrauchsstandards gibt und wie diese wissenschaftlich zu erforschen und zu beschreiben sind. Im ersten Abschnitt wird kurz der Forschungsstand erläutert und die These vertreten, dass man im Deutschen sprechsprachliche, gewissermaßen schriftferne Sprachgebrauchsstandards annehmen muss und dass man diese zielgerichtet untersuchen und beschreiben sollte. Im zweiten Abschnitt werden einige Beispiele für Merkmale der sprechsprachlichen Standards exemplarisch vorgestellt, um deutlich zu machen, welche Beschreibungsaufgaben sich ergeben. Danach wird die Frage erörtert, wie viele bzw. welche Gebrauchsstandards für das Deutsche in Deutschland unterschieden werden können/müssen - dies auch aufgrund von Anregungen vonseiten der Sprachdidaktik im Bereich Deutsch als Fremdsprache (Abschnitt 3). Im vierten Abschnitt handelt es sich um die Forschungs- und Beschreibungsaufgaben, wie sie in Bezug auf diese Frage im Institut für Deutsche Sprache gesehen werden und wie sich am IDS laufende Vorhaben in den Forschungskontext einfügen.
\end{abstract}

\section{Gibt es regionale Gebrauchsstandards?}

Ich möchte diese Frage mit ,ja“ beantworten. Ich gehe davon aus, dass im gegenwärtigen Entwicklungsstadium des Deutschen Sprachformen existieren, die als „regionale Gebrauchstandards“ aufgefasst werden können. Darunter sind geographisch definierte Varietäten- und Sprachgebrauchsmuster zu verstehen, die im jeweiligen regionalen Kontext ein entsprechend hohes Prestige tragen und die sowohl im informellen als auch im formellen Sprachgebrauch angemessen sind und akzeptiert werden. Sie weisen auf allen Sprachebenen spezifische regionaltypische Formen auf und unterscheiden sich von Dialekten und Umgangssprachen einerseits und von der kodifizierten Schriftund Standardsprache andererseits. ${ }^{1}$ Diese Gebrauchsstandards sind zunächst

\footnotetext{
* Dieser Vortrag entstand im Rahmen des IDS-Projekts „Variation des gesprochenen Deutsch" unter Mitarbeit von Stefan Kleiner und Ralf Knöbl.

1 Umgangssprache z. B. in der Definition von Mihm (2000), S. 2107; Standardsprache z. B. in der Definition des Duden, vgl. Duden-Aussprachewörterbuch (2000, Vorwort).
} 
in zwei verschiedenen Dimensionen zu sehen: erstens einer geographischen oder horizontalen und zweitens einer vertikalen Dimension. Bezüglich der horizontalen Dimension gehe ich im Anschluss an Wolf (1994) ${ }^{2}$ davon aus, dass das Deutsche in erster Linie weniger als eine plurinationale (Ammon 1995), sondern vielmehr als eine pluriareale Sprache zu betrachten ist und dass auch innerhalb der deutschsprachigen Länder mehrere Sprachlandschaften zu differenzieren und zu untersuchen sind. ${ }^{3}$ Unter dem Gesichtspunkt der vertikalen Dimension sind die regionalen Gebrauchsstandards im Rahmen der Variations- und Varietätenlinguistik zu sehen. Es herrscht diesbezüglich in der deutschen Sprachwissenschaft ein weit verbreitetes traditionelles Betrachtungsschema, das von einer grundlegenden vertikal ausgerichteten Dichotomie „Hochsprache - Dialekt“ ausgeht, mit einer dazwischen liegenden Schicht, die als Umgangssprache, Regionalsprache oder auch als (neuer) Substandard (Bellmann 1983) angesehen wird; eine typische Veranschaulichung dieses Modells findet sich z.B. im dtv-Atlas (vgl. König 1996).

Man hat sich mit regionaler Variation in der Forschung bisher vorzugsweise mehr oder weniger nur in Bezug auf die „unteren“ Schichten der vertikalen Skala - also mit Dialekten und Umgangssprachen beschäftigt. In der letzten Zeit sind einige Publikationen erschienen, die die regionale Variation explizit in Bezug auf die Standardsprache untersuchen. Besonders im Zusammenhang mit der Diskussion um die Plurizentrizität des Deutschen ist die Variation in der Standardsprache etwas mehr in den Blickpunkt der Forschung gerückt (vgl. z. B. Wiesinger 1988; Ammon 1995; Polenz 1999). In der jüngeren Forschung versucht man, die regionale Variation im Rahmen von Gebrauchsstandards zu sehen. So hat Ammon (1995) im Rahmen seines Konzepts der Plurinationalität des Deutschen auch Gebrauchsstandards konzipiert. Er nutzt das Kriterium der Kodifizierung, um den Gebrauchsstandard von der jeweiligen nationalen Standardvarietät abzugrenzen. Die nichtkodifizierten Varianten, die in Modelltexten wie z. B. überregionalen Nachrichtensendungen vorkommen, stellen den „Gebrauchsstandard“ dar. Diese Kennzeichnung soll implizieren, dass bestimmte Formen im Sprachkodex nicht als standardsprachlich ausgewiesen sind. Ammon unterscheidet demnach im Rahmen der Modelltexte verschiedene Schichten des Standards: den kodifi-

${ }^{2}$ Wolf (1994) schreibt dazu: ,Vorerst scheint es mir günstiger und adäquater zu sein, das Deutsche als eine pluriareale Sprache zu bezeichnen" (S. 74). Er plädiert dafür, auch innerhalb einzelner deutschsprachiger Länder mehrere Sprachlandschaften zu differenzieren.

${ }^{3}$ Dies ist gerechtfertigt insbesondere auch in Bezug auf die Sprecherperspektive. Denn auch Ulrich Ammon geht offensichtlich davon aus, dass gegenwärtig das regionale Sprecherbewusstsein (noch?) stärker bzw. wichtiger ist als das nationale (vgl. Ammon 1995, S. 505-511). 
zierten Standard (ausgewiesen im Sprachkodex als standardsprachlich) und den nichtkodifizierten Standard (Gebrauchsstandard). Der Gebrauchsstandard umfasst nach Ammon teilweise „Sektoren der Sprache, für die es noch gar keine unumstrittenen nationsweiten Varianten gibt, wo also der Prozess der Standardisierung im Sinne der überregionalen Vereinheitlichung nicht zum Abschluss gebracht ist" (Ammon 1995, S. 85).

Auer (1997) stellt Überlegungen zur regionalen Variation im Standarddeutschen in Deutschland an und postuliert bestimmte Entwicklungen in Richtung Destandardisierung, die die Existenz regionaler Standards zur Folge haben: „Tatsächlich muss man wohl davon ausgehen, dass es so etwas wie eine bairische, schwäbische oder auch Hamburger Variante des Standarddeutschen gibt, die im jeweiligen régionalen Kontext das höchste Prestige trägt und die in allen offiziellen Situationen angemessen ist. Daneben existiert in Deutschland allerdings auch weiterhin eine diese überspannende allgemeindeutsche normative Hochsprache; in der Phonetik umfasst sie die Orthoepie, also eine Aussprachenorm, die keiner Region zugeordnet werden kann" (Auer 1997, S. 136). Auer nimmt an, dass die Orthoepie in den letzten Jahren an Bedeutung verloren habe und dass ihre Bedeutung gegenwärtig recht gering sei - ,ihr zu folgen, ist eigentlich nur (noch) für Nachrichtensprecher sinn- und prestigevoll, weder für Politiker, Journalisten oder Universitätsprofessoren, noch für Schauspieler“" (S. 136). Da die Regionalstandards durch den orthoepischen Standard (und somit durch die Schriftsprache) immer noch überdacht sind, beschränkt er die Unterschiede zwischen den Regionalstandards und der Orthoepie auf subphonematische Prozesse wie z. B. die Durchführung der Auslautverhärtung, die Verstimmhaftung von Lenes, die Aspiration bzw. auf die Variationsbreite bei der phonetischen Realisierung der einzelnen Phoneme. Die dabei angedeutete Variationsbreite der Regionalstandards umfasst somit in etwa die regionalen Aussprachenormen, die im Atlas zur Aussprache des Schriftdeutschen in der Bundesrepublik Deutschland beschrieben sind (König 1989).

Eichinger (2001) beschreibt einen regionalen Gebrauchsstandard (am Beispiel des Süddeutschen) explizit nicht als Subsystem (wie z. B. ein Dialekt, aber auf höherer Ebene), sondern als „Varietäten- und Sprachgebrauchsmuster, das sich durch eine spezifische Auswahl aus den Optionen auszeichnet, die im deutschen Sprachraum zur Verfügung stehen“ (2001, S. 62). Er zieht daraus den methodologischen Schluss, dass es bei der Beschreibung von regionalen Gebrauchsstandards nicht darauf ankomme, sie vollständig zu beschreiben, sondern es wäre die Angabe einer Reihe von „,auffälligen, salienten Merkmalen“" (S. 62) nötig. In der Phonetik beschränkt er sich bei der Angabe der salienten Merkmale in etwa auf die regionalen Ausspracheformen von König (1989).

Diese Ansätze stellen Versuche dar, die im traditionellen Betrachtungsschema vorausgesetzte, nicht-realistische regionale Homogenität des Standards zu überwinden und dadurch seiner tatsächlichen sprachlichen Realität 
näher zu kommen. Insgesamt jedoch kann meines Erachtens festgehalten werden, dass die Autoren in dieser Intention nicht weit genug gehen, und zwar deswegen, weil sie ihren Betrachtungen einen doch immer noch relativ engen, eher schriftsprachorientierten Standardbegriff zugrunde legen und das tatsächlich gesprochene Register der Standardsprache nicht berücksichtigen. Diesem traditionell eng gefassten Standardbegriff entspricht nämlich die gerade im deutschen Sprachraum verbreitete Auffassung, dass alles, was vom schriftsprachlichen Standard abweicht, bereits Umgangssprache - mit reduziertem Prestige versehen - sei. ${ }^{4}$ Nach dieser Auffassung liegt die Umgangssprache außerhalb der akzeptierten Grenzen der Standardvarietät. ${ }^{5}$

Demgegenüber plädiere ich für einen erweiterten Standardbegriff, der es erlaubt, auch verbreiteten bzw. gängigen sogenannten umgangssprachlichen Phänomenen eine normative Geltung, d.h. eine Standardqualität zuzuschreiben. Ich würde es deshalb für sinnvoll halten, auch in Bezug auf das Deutsche von formellen und informellen Standards zu sprechen, so wie es der englische Germanist Martin Durrell z.B. für das Englische konstatiert und für das Deutsche vorschlägt. Durrell (1998, S. 303) weist auf die äußerst ,restriktive Vorstellung von Standardsprache" im deutschsprachigen Raum hin. Diese würde im krassen Unterschied z. B. zum Englischen oder Französischen stehen. Im Englischen existiere eine ,alltägliche britische Sprechsprache“ (Durrell 2003, S. 250), die als „Colloquial English“ bzw. informelles Register auch zum Standardenglischen gehöre; diese Sprachform werde als informeller Sprechstandard selbstverständlich auch im Fremdsprachenunterricht berücksichtigt. Die Lerner werden mit typischen Merkmalen der alltäglichen britischen Sprechsprache in völlig natürlichen Gesprächen konfrontiert und es wird im Unterricht auch konsequent auf relevante Unterschiede zwischen dem formellen und informellen Register des Englischen hingewiesen. So werden auch die in der geschriebenen Sprache verpönten mündlichen Ausdrücke wie z. B. die Konstruktion have got gelehrt (Durrell 2003, S. 250). - Im Deutschen dagegen existiere eine ganz andere Einstellung gegenüber der Sprachform, die gelehrt werden muss: Das sollte die Schriftsprache sein oder eine an die Schriftsprache eng angelehnte schriftnahe, schriftabhängige mündliche Form. Die Existenz einer Sprachform wie „Colloquial German“ - oder einer „alltäglichen deutschen Sprechsprache“ - wird im Deutschen nach den Erfahrungen von Martin Durrell und anderen ausländischen Sprachgermanis-

${ }^{4}$ Diese Meinung ist weit verbreitet. Vgl. z. B. die Anmerkung im Duden-Aussprachewörterbuch (2000, S. 64) bezüglich des Anwendungsbereichs der Umgangslautung: „Die Umgangslautung herrscht ... in der gewöhnlichen Unterhaltung zu Hause, auf der Straße und im Betrieb vor und wird für die Wiedergabe sprachlich und inhaltlich weniger anspruchsvoller Texte verwendet".

5 Vgl. dazu ausführlich Durrell (1995), (1999) und (2003) mit den entsprechenden bibliographischen Angaben zu diesem Problembereich. 
ten konsequent verleugnet ${ }^{6}$, zumindest in den Lehrmaterialien Deutsch als Fremdsprache. So vertreten z. B. auch Barbour/Stevenson (1998) folgende Meinung: „Während die von Quirk und Greenbaum verfasste Grammatik des Englischen eine realistische Beschreibung der modernen Sprachform gebildeter Muttersprachler verkörpert, sucht man in deutschen Grammatiken bis heute vergebens nach Verweisen auf bestimmte, auch in der Sprechweise gebildeter Leute anzutreffende Erscheinungen" (S. 147). Davon, dass diese Auffassungen zutreffen, kann man sich überzeugen, indem man sich beispielsweise einmal die Standarddefinition des Duden-Aussprachewörterbuchs vor Augen führt: Danach wird die Standardlautung als eine überregionale, einheitliche, schriftnahe und deutliche Gebrauchsnorm konzipiert (Duden-Aussprachewörterbuch 2000, Vorwort). Eine solche Einstellung betrachtet Durrell (1993) aus der Sicht des Unterrichts Deutsch als Fremdsprache als ein besonderes Problem, denn das Deutsche wird als eine sehr formelle und variationslose Sprache „ohne anerkannte alltagssprachliche Variation" dargestellt, „deren Normen mit außerordentlicher Strenge verteidigt wurden“ (S. 424). Das Problem kommt nach seiner Meinung zu selten zur Sprache und wird zu wenig berücksichtigt.

Ich möchte noch einmal kurz zu charakterisieren versuchen, was ich unter „regionalen Gebrauchsstandards“ verstehen will - und zwar aus der Sicht eines Deutschlerners, also einer Perspektive, wie sie Martin Durrell in den zitierten Aufsätzen einnimmt. Wenn sich ein Deutschlerner über die Sprechsprache in Deutschland informieren will, dann stößt er auf Wörterbücher und andere Hilfsmittel, die ihm eine schriftnahe Sprech- bzw. Aussprachenorm anbieten. Zum Beispiel, dass die Artikelform eine als [ainə] gesprochen wird. Kommt der Deutschlerner nach Deutschland, erfährt er aber etwas anderes, nämlich, dass die oben angegebene schriftnahe Form eine im Alltagsgebrauch nur wenig oder gar nicht verwendet wird. In Norddeutschland mit Ausbreitungstendenz nach Süden wird nämlich nicht [ainə] gesprochen, sondern [rəə]. In Bayern wird statt [ainə] noch [a] gesprochen; [nə] und [a] sind somit die tatsächlichen Gebrauchsformen, die aber in den traditionellen Wörterbüchern aufgrund der deutschen Lexikographiegeschichte nicht verzeichnet werden und auch in Lehrbüchern nicht erscheinen.

Diese Auffassung von regionalen Gebrauchsstandards unterscheidet sich von den oben skizzierten Positionen, die alle mit einem relativ engen, schriftsprachorientierten Standardbegriff operieren. Dabei verstehe ich die regionalen Gebrauchsstandards nicht nur als regionale Aussprachenorm im Sinne eines sehr formellen Standardbegriffs, sondern auch als Muster, die im sprech-

${ }^{6}$ Vgl. die Äußerung von Durrell (2003) dazu: „Obwohl charakteristische Merkmale des gesprochenen Registers in einigen Hörverständnisdialogen vorkommen, wird die Existenz dieser Formen effektiv dadurch vertuscht, dass der Lerner diese Texte nicht sieht und dass nicht explizit auf etwaige Unterschiede zur Schrift- und Hochsprache hingewiesen wird" (S. 251). 
sprachlichen nichtformellen Register wirksam sind. Die Sprecher treffen eine standardsprachliche Auswahl aus lautlichen, lexikalischen, morphologischen und syntaktischen Varianten gemäß den regionalen Mustern bzw. Normen in den entsprechenden sprachlichen Situationen.

\section{Beispiele für Merkmale von sprechsprachlichen Regionalstandards}

Anhand einiger ausgewählter Beispiele und Karten möchte ich im Folgenden das Konzept der regionalen Gebrauchsstandards etwas näher erläutern. Einige prototypische Beispiele der regionalen Gebrauchsstandards sind in den Tabellen 1-3 zusammengestellt. ${ }^{7}$ Diese Beispiele stammen größtenteils ${ }^{8}$ aus einem Korpus, das von Werner König Mitte der 1970er Jahre für die Untersuchung der regionalen Variation im Deutschen erhoben wurde. Im Atlas zur Aussprache des Schriftdeutschen in der Bundesrepublik Deutschland, der 1989 erschienen ist, stellt König für die von ihm analysierte Vorlesesprache Folgendes fest (vgl. König 1989, Bd. 1, S. 122):

„Die Ergebnisse demonstrieren, dass es keine Region in der Bundesrepublik gibt, deren Aussprache den Regularitäten eines der Wörterbücher (auch in den „gemäßigten“ Varianten, „Umgangslautung“) voll entspricht. Das Bild, das die Karten zeigen, ist vielmehr so vielfältig, dass es weiterer intensiver Analysen bedarf zur Klärung der Frage, wo in der Bundesrepublik das beste „Hochdeutsch“ gesprochen wird und welches der Aussprachewörterbücher in seiner Normierung der Realität gebildeter Sprecher am nächsten kommt".

Die Analysen der regionalen Variation im Standarddeutschen werden gegenwärtig im IDS durchgeführt, indem der spontansprachliche Teil des von König erhobenen flächendeckenden ${ }^{9}$ Korpus im Projekt „Variation des gesprochen Deutsch“ ausgewertet wird. ${ }^{10}$ Dieses Korpus besteht aus Gesprächen und Interviews mit Studenten/Akademikern aus 43 verschiedenen Herkunftsorten der (alten) Bundesrepublik Deutschland. ${ }^{11}$ Analysiert wurden bisher ca. zehnminütige Abschnitte aus jeder Aufnahme (insgesamt etwa 7 Tonbandstunden, ca. 37.000 Tokens), und zwar Merkmale, die eine hohe Gebrauchsfrequenz aufweisen. Um die Signifikanz der regionalen Verteilung zu überprüfen, wurde die Kartiermethode angewendet. Der Vorteil dieses Korpus z. B. im Vergleich zum Pfeffer-Korpus - besteht darin, dass es sich nicht um in gewisser Weise zufällige Aufnahmen handelt, sondern dass es methodisch

${ }^{7}$ Die in den Tabellen 1-3 angeführten Beispielsätze sind durchgehend nummeriert.

${ }^{8}$ Sie enthalten auch einige Beispiele aus dem öffentlichen Sprachgebrauch bekannter Politiker, und zwar die Beispiele (5), (6), (7), (25), (33), (43), (44).

${ }^{9}$ Nur die frühere Bundesrepublik Deutschland. Die anderen deutschsprachigen Länder Österreich und die Schweiz sowie die frühere DDR wurden leider nicht erfasst.

${ }_{10}$ Zum Projekt s. http:/ www.ids-mannheim.de/prag/AusVar/

11 Zur ausführlichen Darstellung der Erhebung vgl. König (1989) und König (1996). 
kontrolliert erhoben wurde und daher einen repräsentativen Schnitt des informellen Registers gebildeter Sprecher dokumentiert.

Ich unterscheide im folgenden zwei Typen von Merkmalen der regionalen Gebrauchsstandards: 1) sprechsprachliche und 2) regionaltypische. Unter sprechsprachlichen Merkmalen sind zunächst zu verstehen sehr verbreitete und allgemein bekannte Merkmale des gesprochenen Deutsch (vgl. Tabelle 1). Dazu gehören u.a. typische Reduktionsformen, die im untersuchten Material besonders häufig vorkommen, wie die schwachen Formen (sog. weak forms) von Artikeln, Pronomen, Adverbien, Konjunktionen usw., die in unbetonten Positionen auftreten und verschiedenen Tilgungen bzw. Auslassungen, Apokopen, Elisionen oder Assimilationen unterworfen sind. Man geht im allgemeinen davon aus (so z. B. Kohler 1995), dass solche Phänomene überregionale Merkmale des gesprochenen Deutsch allgemein darstellen. Einige prototypische Beispiele für sprechsprachliche Phänomene sind in der folgenden Tabelle dargestellt.

Tab. 1: Sprechsprachliche Varianten ${ }^{12}$

\begin{tabular}{|c|c|c|}
\hline \multicolumn{2}{|c|}{ Beispiele } & \multirow{2}{*}{$\begin{array}{l}\text { Variationsphänomene } \\
\text { <eine> eine-ne-ale- } \\
\text { Variation }\end{array}$} \\
\hline (1) & $\begin{array}{l}\text { Freiburg: „Ich mein so Leute aus-m Ruhrgebiet, } \\
\text { die ham ne/ne ziemlich harte Sprache“" } \\
\text { Augsburg: „So des war } \boldsymbol{a} \text { Klass nach uns, glaub ich“ }\end{array}$ & \\
\hline (3) & $\begin{array}{l}\text { Stuttgart: „Also ich ich erinner mich an die Volkschul } \\
\text { nich mehr, aber ans Gymnasium auf keinen Fall“ }\end{array}$ & $\begin{array}{l}<\text { nicht }> \\
\text { nicht }- \text { nich - net- } \\
\text { Variation }\end{array}$ \\
\hline (4) & $\begin{array}{l}\text { Karlsruhe: „Ich weiß es also wirklich net, wo-s } \\
\text { herkommt" }\end{array}$ & \\
\hline (5) & $\begin{array}{l}\text { „Du darfscht die Mauer net bauen, du darfscht den } \\
\text { Arafat nicht ausweisen“ (K. Kinkel in Phönix) }\end{array}$ & \\
\hline (6) & $\begin{array}{l}\text { „Wir sollten miteinander reden, und zwar net } \\
\text { öffentlich“ (A. Nahles im } Z D F \text { ) }\end{array}$ & \\
\hline (7) & $\begin{array}{l}\text { „Ich versteh den Satz net, muss ich ehrlich sagen“" } \\
\text { (H. Seehofer bei Christiansen) }\end{array}$ & \\
\hline (8) & $\begin{array}{l}\text { Nördlingen: „Mei Mutter is Lehrerin, ja“; } \\
\text { Kiel: „Ja, aber bei mir is-es tatsächlich erst die zweite } \\
\text { Generation“ }\end{array}$ & $\begin{array}{l}<\mathrm{ist}> \\
i s t-i s-i s c h\end{array}$ \\
\hline (9) & $\begin{array}{l}\text { Stuttgart: „Und zwar, isch ihnen irgendwas aufge- } \\
\text { fallen in/an meim Reden, außer dem (...)“ }\end{array}$ & \\
\hline (10) & $\begin{array}{l}\text { Bayreuth: „Wir sin insgesamt in unserem Jahrgang } \\
\text { so - jetz noch hundertzwanzich Studenten, anfangs } \\
\text { waren-s hundertfünfzig“ }\end{array}$ & $\begin{array}{l}<\text { jetzt }>\text {, <und }> \\
{[\mathrm{t}]-\text { Tilgung }(\text { jetz, un })}\end{array}$ \\
\hline
\end{tabular}

12 Lexeme, die das fokussierte Variationsphänomen veranschaulichen, sind mit Fett- und Kursivschrift hervorgehoben. 
Fortsetzung Tabelle 1

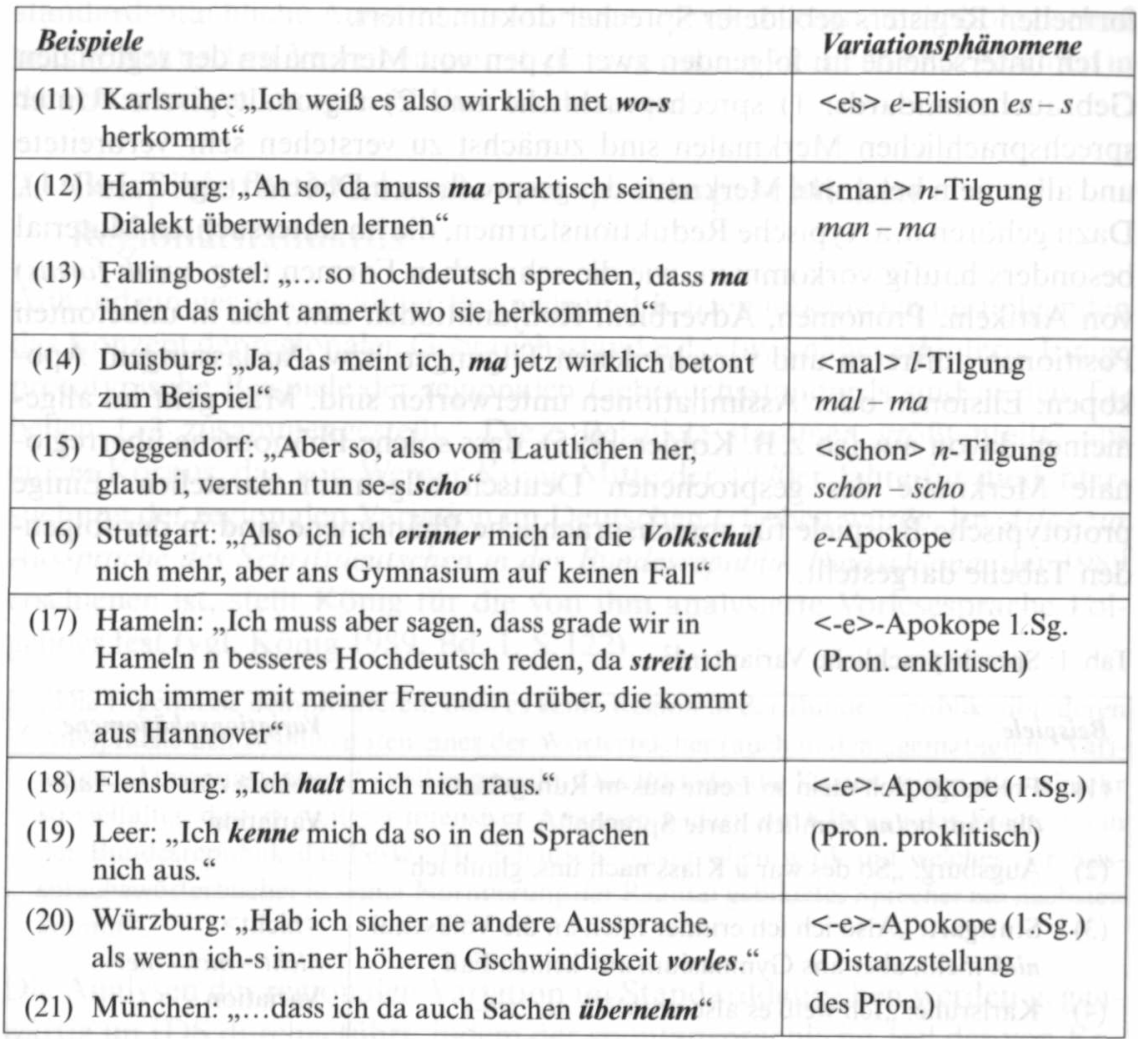

Unter regionaltypischen Merkmalen (vgl. Tab. 2) sind dagegen zunächst solche zu verstehen, die nicht als überregionale Merkmale der gesprochenen deutschen Sprache allgemein angesehen werden können, sondern solche, die geographisch bedingt sind. Die Ausprägung dieser Varianten - d.h. zum Beispiel ihre Form und ihr grundsätzlicher arealer Geltungsbereich - sind in der Dialektologie und in der Regionalspracheforschung (Mihm 2000) im Allgemeinen bereits bekannt. Bei der Untersuchung der Regionalstandards kann daher von bestimmten Gebrauchserwartungen in Bezug auf diese Merkmale in bestimmten Regionen ausgegangen werden. 
Tab. 2: Regionaltypische Merkmale

\begin{tabular}{|c|c|c|}
\hline \multicolumn{2}{|c|}{ Beispiele } & \multirow{3}{*}{$\begin{array}{l}\text { Variationsphänomene } \\
<a ̈> \\
{[\mathrm{e}:] /[\varepsilon:]-\text { Variation }}\end{array}$} \\
\hline (22) & $\begin{array}{l}\text { Duisburg: „Aber ich hab n Freund, der is aus Mün- } \\
\text { chen, un der lacht also immer da drüber, wenn wir zum } \\
\text { Beispiel „,Käse“, wir sprechen das „ä“ so ganz breit. } \\
\text { Das is für uns Hochdeutsch un die sagen „Keese““" }\end{array}$ & \\
\hline (23) & $\begin{array}{l}\text { München: „Erstens mal versteh ich-n [den Augsburger } \\
\text { Dialekt] nich/ also relativ/ wenn er so richtich } \\
\text { ausgepreegt gsprochen wird - schlecht“ }\end{array}$ & \\
\hline (24) & $\begin{array}{l}\text { Fallingbostel: „Meistens is ja Ostern, Fingsten, da } \\
\text { is-s Semester zu Ende“" }\end{array}$ & $\begin{array}{l}<\mathrm{pf}-> \\
{[\mathrm{pf}] /[\mathrm{f}]-\text { Variation }}\end{array}$ \\
\hline (25) , & $\begin{array}{l}\text { „Flegeversicherungsbeitrag" [Pflege...] (U.Schmidt); } \\
\text { „Fandflaschen“ [Pfand...] (J. Trittin) }\end{array}$ & \\
\hline (26) & $\begin{array}{l}\text { Wittlich: „Immer, wenn isch, wenn isch längere Zeit zu } \\
\text { Hause war und komm dann wieder hierhin,... }\end{array}$ & $\begin{array}{l}<\mathrm{ch}> \\
{[\mathrm{c}] /\left[\int\right]}\end{array}$ \\
\hline (27) & $\begin{array}{l}\text { Koblenz: „Mansche Leute, die gleischen sisch ja so n } \\
\text { bissn der Sprache, die hier gesprochen wird, an“ }\end{array}$ & Koronalisierung \\
\hline (28) & $\begin{array}{l}\text { Nordhorn: „Und - das waren Marburch, Würzburch, } \\
\text { Freiburch. Und die hab ich angeschrieben. Ich bekam } \\
\text { aus Marburg eine Zusage, aus Freiburg eine Zusage } \\
\text { und, ähm, Würzburg auch" }\end{array}$ & $\begin{array}{l}<-r g>\text { (Spirantisierung) } \\
{[\mathrm{rk}] /[\mathrm{rc}]-\text { Variation }}\end{array}$ \\
\hline (29) & $\begin{array}{l}\text { Bremerhaven „Ja, ich würd schon sagen, das es } \\
\text { hochdeutsch ist mit eim gewissen Einschlach" }\end{array}$ & $\begin{array}{l}<-\mathrm{g}>\text { Spirantisierung } \\
{[\mathrm{k}] /[\mathrm{x}]-[\mathrm{c}]-\text { Variation }}\end{array}$ \\
\hline (30) & Bayreuth: „hundertzwanzich ... hundertfïnfzig“" & \\
\hline (31) & $\begin{array}{l}\text { Bremen: „Aber das is also sehr ausgepreecht bei man- } \\
\text { chen Leuten, die zum Beispiel aus Bremen kommen“ }\end{array}$ & $\begin{array}{l}<-\mathrm{gt}> \\
{[\mathrm{kt}] /[\mathrm{xt}]-[c ̧ t]}\end{array}$ \\
\hline (32) & $\begin{array}{l}\text { Nürnberg: „Ich mein, ich bin runterkommen (und) } \\
\text { hab glei eins } \text { kricht }^{\prime \prime}\end{array}$ & $g$-Spirantisierung \\
\hline (33) & $\begin{array}{l}\text { „Mir liecht } \text { daran“ (Kanzler Schröder); „Die Sache } \\
\text { beweecht mich“ (F. Merz); „... treecht kein Kopftuch“ } \\
\text { (M.-L. Beck) }\end{array}$ & \\
\hline (34) & $\begin{array}{l}\text { Augsburg: „Unser Professoren hier zumindescht } \\
\text { strengen sich sehr an“" }\end{array}$ & $\begin{array}{l}<\mathrm{st}> \\
{[\mathrm{st}] /[\mathrm{ft}]}\end{array}$ \\
\hline$(35)$ & $\begin{array}{l}\text { Offenburg: „Ja, ich mein, bewusst acht ich fascht } \\
\text { immer auf meine Sprache“ }\end{array}$ & $s$-Palatalisierung \\
\hline (36) & $\begin{array}{l}\text { Stuttgart: „Also ich war mal n Semeschter an der PH } \\
\text { un da hab ich ... }\end{array}$ & \\
\hline & $\begin{array}{l}\text { Würzburg: „Hab ich sicher ne andere Aussprache, } \\
\text { als wenn ich-s in-ner höheren Gschwindigkeit vorles“ }\end{array}$ & $\begin{array}{l}<\mathrm{ge}-> \\
{[\mathrm{g} \partial] /[\mathrm{g}]}\end{array}$ \\
\hline (38) & $\begin{array}{l}\text { Kempten: „Des Jahr, wenn ich deutsch gsprochen hab, } \\
\text { ham die Schüler also am Anfang ganz dumm gschaut, } \\
\text { weil die ham einfach den süddeutschen Akzent ghört } \\
\text { und da/ des waren sie net gwöhnt“ }\end{array}$ & $e$-Synkope \\
\hline
\end{tabular}


Im Folgenden werden einige Merkmale von regionalen Gebrauchsstandards etwas näher am Beispiel von Sprachkarten erläutert.

Einige Vorbemerkungen zum Verständnis der Karten:

- Links oben ist das Variationsphänomen angegeben, das auf der Karte beschrieben wird: [,eine“, unbest. Artikel] - Gebrauch des unbestimmten Artikels „eine“ im Korpus.

- In dem Kasten rechts oben sind die im Korpus belegten Varianten des Artikels angegeben: eine - ne-ale. Für jede Variante ist die Vorkommenshäufigkeit (in Prozent) mit Hilfe von „Tortensymbolen“ dargestellt.

- Die Symbole sind farblich folgendermaßen abgestimmt: standard- und schriftnähere Formen (,eine“) werden durch helleren Grauton dargestellt; die regional-sprechsprachlichen Varianten sind durch dunklere Grautöne („ne“) bzw. durch schwarze Symbole (,a/e“) dargestellt.

Die Karte eine (vgl. Abb.1). beschreibt den Gebrauch des unbestimmten Artikels „eine“. In unserem Korpus sind insgesamt drei Varianten belegt, wie der Legende zu entnehmen ist: die wörterbuchgemäße Artikelform ,eine“ und zwei sprechsprachliche Varianten, die in Wörterbüchern nicht vorkommen die Reduktionsform „ne“ und die vokalische Variante „a“. In Bezug auf die schriftbasierte Form „eine“ ist festzustellen, dass sie eine nur geringe $\mathrm{Ge}$ brauchsfrequenz aufweist (insgesamt 25 Prozent der Belege) und daher u.E. nicht als sprechsprachliche Norm angesehen werden kann. In Bezug auf die beiden sprechsprachlichen Formen des Artikels gibt es großräumige regionale Unterschiede im Gebrauch. Die Form ,ne“ ist die häufigste und großräumig verwendete im Korpus (sie macht 60 Prozent der Belege aus) und kann daher als sprechsprachliche schriftferne Hauptvariante in diesem Korpus angesehen werden. Sie wird aber in Bayern nicht verwendet (Ausnahmen Nördlingen und Simbach mit je 10 Prozent). In Bayern bzw. im Südosten wird die vokalische Form ,a" für ,eine“ verwendet, die dort 80 Prozent der Belege darstellt und somit als stabiles Merkmal des regionalen Standards angesehen werden kann. Die Karte zeigt deutlich, dass keine einheitliche Südnorm bezüglich des Gebrauchs der sprechsprachlichen Varianten existiert. Im Wortatlas der deutschen Umgangssprachen (Eichhoff 2000) ${ }^{13}$ liegt für die Formen „,ne“ und „a“ noch eine klare Nord-Süd-Gliederung vor. Belege für „ne“ finden sich bei Eichhoff im Süden nur vereinzelt in Hessen und Baden-Württemberg (Frankfurt und Freiburg), ansonsten ist überall im Süden vokalisches „a“ belegt. Man könnte also nach dem König-Korpus von einer Expansion der Form „ne“ in den südwestlichen Raum sprechen.

${ }^{13}$ Eichhoff (2000), Karte 4-66, im Satz „Er hat sich eine Wiese gekauft“. 


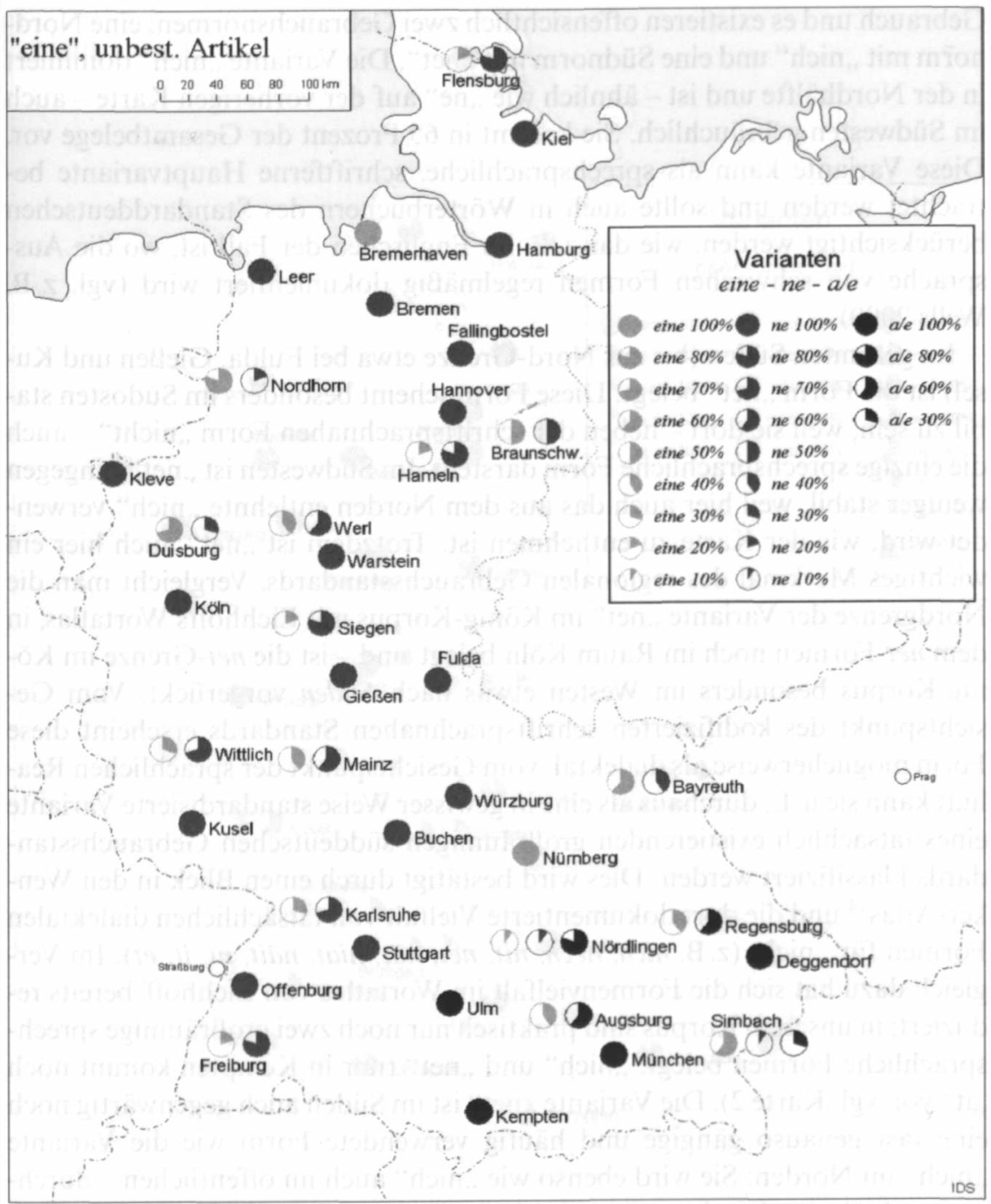

Abb. 1: Gebrauchsvarianten des Artikels eine

Auf der zweiten Karte geht es um die Formen von „nicht“ (vgl. Abb. 2). In unserem Korpus sind neben der schriftbasierten wörterbuchgemäßen Form „nicht" noch zwei sprechsprachliche Hauptvarianten belegt: „nich“ (mit getilgtem $t$ ) und die Variante „net“. Bezüglich der schriftsprachgemäßen Wörterbuchform „nicht" muss festgehalten werden, dass sie zwar im gesamten Untersuchungsgebiet verstreut vorkommt, aber mit einer sehr geringen Gebrauchsfrequenz (7 Prozent aller Belege) und sie kann daher nicht die sprechsprachliche Norm repräsentieren. In Bezug auf die sprechsprachlichen Varianten „nich“ und „net“ zeigen sich großräumige regionale Unterschiede im 
Gebrauch und es existieren offensichtlich zwei Gebrauchsnormen: eine Nordnorm mit „nich“ und eine Südnorm mit ,net“. Die Variante „nich“ dominiert in der Nordhälfte und ist - ähnlich wie „ne“ auf der vorherigen Karte - auch im Südwesten gebräuchlich. Sie kommt in 65 Prozent der Gesamtbelege vor. Diese Variante kann als sprechsprachliche, schriftferne Hauptvariante betrachtet werden und sollte auch in Wörterbüchern des Standarddeutschen berücksichtigt werden, wie das z. B. im Englischen der Fall ist, wo die Aussprache von schwachen Formen regelmäßig dokumentiert wird (vgl. z. B. Wells 2000).

Im gesamten Süden (bis zur Nord-Grenze etwa bei Fulda, Gießen und Kusel) ist die Form „net“ belegt. Diese Form scheint besonders im Südosten stabil zu sein, weil sie dort - neben der schriftsprachnahen Form „nicht“ - auch die einzige sprechsprachliche Form darstellt. Im Südwesten ist „net“ hingegen weniger stabil, weil hier auch das aus dem Norden entlehnte „nich“ verwendet wird, wie der Karte zu entnehmen ist. Trotzdem ist "net" auch hier ein wichtiges Merkmal des regionalen Gebrauchsstandards. Vergleicht man die Nordgrenze der Variante „net“ im König-Korpus mit Eichhoffs Wortatlas, in dem net-Formen noch im Raum Köln belegt sind - ist die net-Grenze im König-Korpus besonders im Westen etwas nach Süden vorgerückt. Vom Gesichtspunkt des kodifizierten schriftsprachnahen Standards erscheint diese Form möglicherweise als dialektal; vom Gesichtspunkt der sprachlichen Realität kann sie u. E. durchaus als eine in gewisser Weise standardisierte Variante eines tatsächlich existierenden großräumigen süddeutschen Gebrauchsstandards klassifiziert werden. Dies wird bestätigt durch einen Blick in den Wenker-Atlas ${ }^{14}$ und die dort dokumentierte Vielfalt von tatsächlichen dialektalen Formen für „nicht (z. B. nich, nech, nit, net, nöt, niat, näit, ni, it, et). Im Vergleich dazu hat sich die Formenvielfalt im Wortatlas von Eichhoff bereits reduziert; in unserem Korpus sind praktisch nur noch zwei großräumige sprechsprachliche Formen belegt: „nich“ und „net“ (nur in Kempten kommt noch „it“ vor, vgl. Karte 2). Die Variante „,net“ ist im Süden auch gegenwärtig noch eine fast genauso gängige und häufig verwendete Form wie die Variante „nich“ im Norden: Sie wird ebenso wie „nich“ auch im öffentlichen - durchaus auch überregionalen - Sprachgebrauch verwendet, wie drei aktuelle Beispiele von bekannten Politikern zeigen:

- aus dem südwestdeutschen Raum (Tab. 1, Satz 5): „Du darfscht die Mauer net bauen, du darfscht den Arafat nicht ausweisen" (K. Kinkel in Phönix); - aus dem südostdeutschen Raum (Tab. 1, Satz 7): „Ich versteh den Satz net, muss ich ehrlich sagen" (H. Seehofer bei Christiansen);

- aus dem rheinland-pfälzischen Raum (Tab. 1, Satz 6: „Wir [mit Bezug auf den Kanzler] sollten miteinander reden, und zwar net öffentlich“ (A. Nahles im $Z D F)$.

14 DiWA (vgl. Bibliographie) 


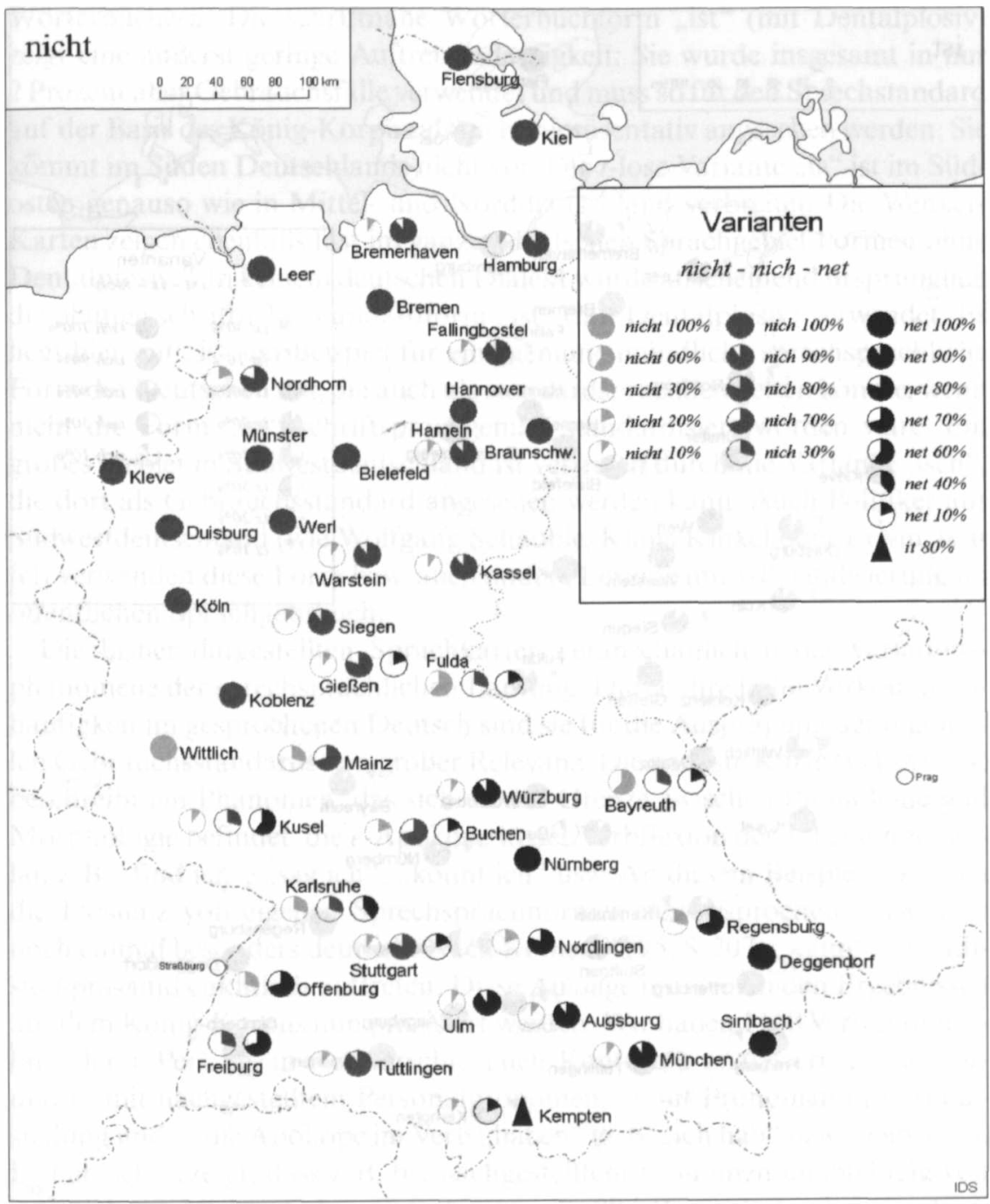

Abb. 2: Gebrauchsvarianten von nicht

Aus den beiden vorgeführten Karten kann bereits abgeleitet werden, dass Bayern allgemein resistenter gegen sprachliche Neuerungen zu sein scheint was möglicherweise etwas mit der Frage der Zukunft der regionalen Gebrauchsstandards zu tun hat. Es hat zumindest die norddeutsche Sprechform „nich" nicht übernommen, genauso wie die Artikelform „ne“ (auf der Karte 1), sondern bleibt bei den eigenen, z. T. standardisierten Formen „net“ und „a“. Dies gilt für den Zeitpunkt der Erhebung des König-Korpus, die Mitte der 1970er Jahre stattgefunden hat. Heutzutage hat sich die sprachliche Situation wohl auch in Bayern teilweise verändert. So thematisiert der För- 


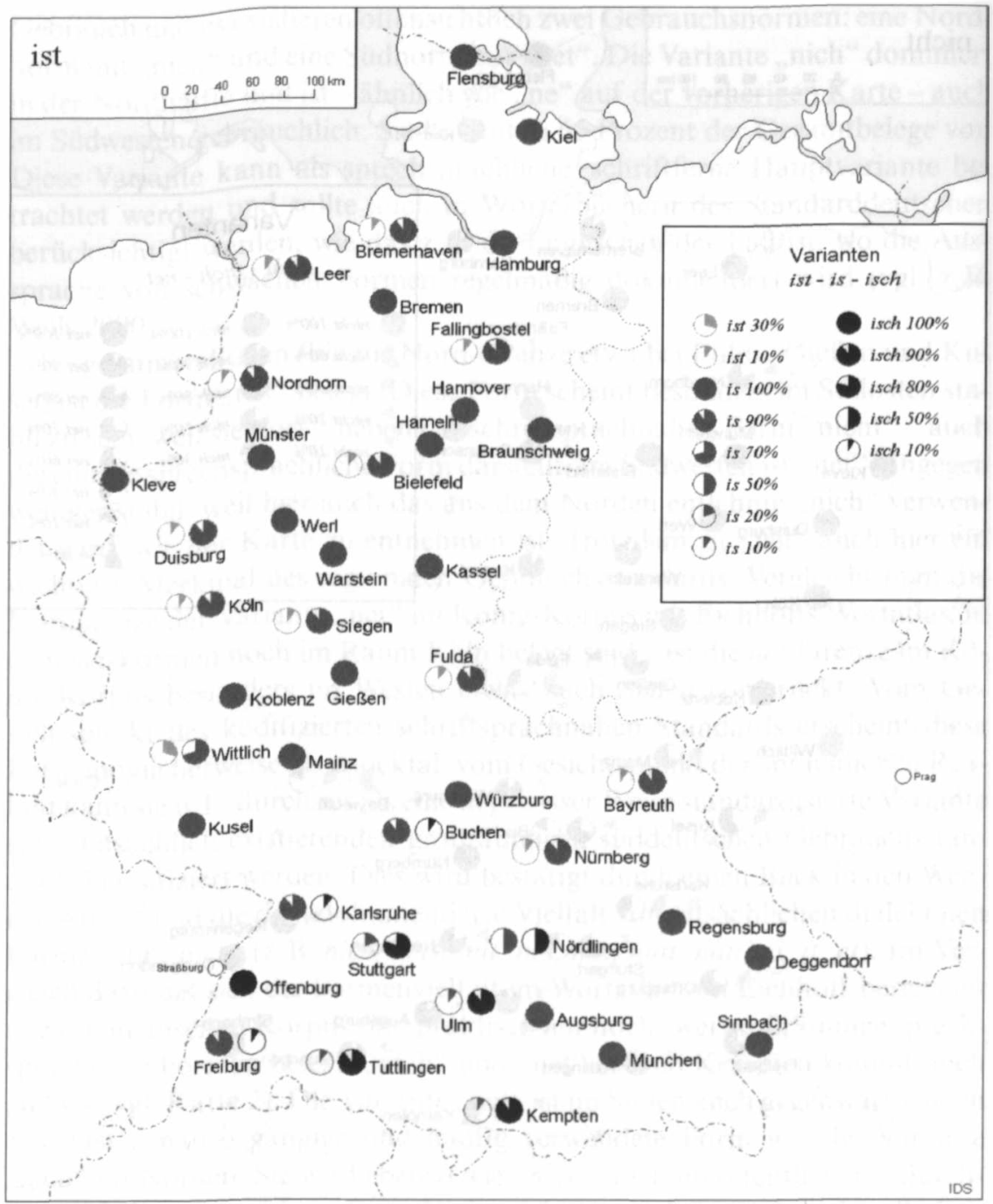

Abb. 3: Gebrauchsvarianten von ist

derverein bairische Sprache und Dialekte ${ }^{15}$ in ganz besonderer Weise die norddeutschen Formen „nich“ und „ne“, die wohl gegenwärtig zumindest in München häufig zu hören sind.

Die Karte 3 beschreibt die Verwendung von „ist“ im Korpus (Abb. 3). Zwei sprechsprachliche Varianten sind in Deutschland im Gebrauch: „is“" (mit getilgtem $t$ ) und „,isch“ mit $s$-Palatalisierung. Beide Formen erscheinen nicht in

${ }^{15}$ Vgl. im Internet: www.bayerische-sprache.de 
Wörterbüchern. Die schriftnahe Wörterbuchform „ist“ (mit Dentalplosiv) zeigt eine äußerst geringe Auftretenshäufigkeit: Sie wurde insgesamt in nur 2 Prozent aller Gebrauchsfälle verwendet und muss so für den Sprechstandard auf der Basis des König-Korpus als nicht repräsentativ angesehen werden. Sie kommt im Süden Deutschlands nicht vor. Die $t$-lose Variante „is“ ist im Südosten genauso wie in Mittel- und Norddeutschland verbreitet. Die WenkerKarten zeigen ebenfalls fast im ganzen deutschen Sprachgebiet Formen ohne Dentalplosiv. ${ }^{16}$ In keinem deutschen Dialekt wurde anscheinend ursprünglich die heutige schriftliche Standardform ,ist“ (mit Dentalplosiv) verwendet. Es liegt hier mit „is“ ein Beispiel für eine genuine mündliche sprechsprachliche Form des Deutschen vor, die auch als standardsprachlich gelten könnte, wenn nicht die Form „ist“ schriftsprachgemäß standardisiert worden wäre. Ein großes Gebiet in Südwestdeutschland ist vertreten durch die Variante „isch“, die dort als Gebrauchsstandard angesehen werden kann. Auch Politiker aus Südwestdeutschland (wie Wolfgang Schäuble, Klaus Kinkel oder Erwin Teufel) verwenden diese Form bzw. auch andere Lexeme mit s-Palatalisierung im öffentlichen Sprachgebrauch.

Die bisher dargestellten Sprachkarten veranschaulichen die Variationsphänomene der sprechsprachlichen Lautung. Durch ihre hohe Vorkommenshäufigkeit im gesprochenen Deutsch sind sie für die Ausprägung der regionalen Gebrauchsstandards von großer Relevanz. Die nächste Karte (vgl. Abb. 4) beschreibt ein Phänomen, das sich an der Grenze zwischen Phonologie und Morphologie befindet: die $e$-Apokope in der Verbflexion der 1. Person Singular, z. B. „find ich“, „sag ich“, „könnt ich“ usw. An diesem Beispiel zeigt sich die Existenz von eigenen Sprechsprachnormen des gesprochenen Deutsch noch einmal besonders deutlich. Nach Kohler (1995, S. 207) „kann“ die $e$-Elision prä- und enklitisch auftreten. Diese Aussage muss nach den Ergebnissen aus dem König-Korpus nun präzisiert werden. Wir haben 1160 Verwendungsfälle der 1.Pers. Sg. in vier verschiedenen Kontexten analysiert: a) mit vorund b) mit nachgestelltem Personalpronomen, c) mit Pronomen in Distanzstellung und d) die Apokope im Verb „haben“ (z. B. ,ich hab“ bzw. „hab ich“). Es hat sich gezeigt, dass z. B. bei nachgestelltem Pronomen unabhängig von regionalsprachlichen Faktoren fast immer (in 98,8 Prozent) Apokope eintritt. Hier könnte also bereits eine neue sprechsprachliche Regel formuliert werden, die den Gebrauch der Personalendung in der Verbflexion der 1. Sg. in der Sprechsprache beschreibt. In einigen Grammatiken wird zwar auf die Variabilität als solche hingewiesen ${ }^{17}$, aber in keiner Grammatik des Deutschen findet sich diese fast zu 100 Prozent gültige Regel des gesprochenen Deutsch, die folglich auch im DaF-Unterricht berücksichtigt werden sollte.

${ }^{16}$ DiWA (vgl. Bibliographie)

17 Eine ausführliche Übersicht dazu s. Eisenberg (1998), S. 182. 


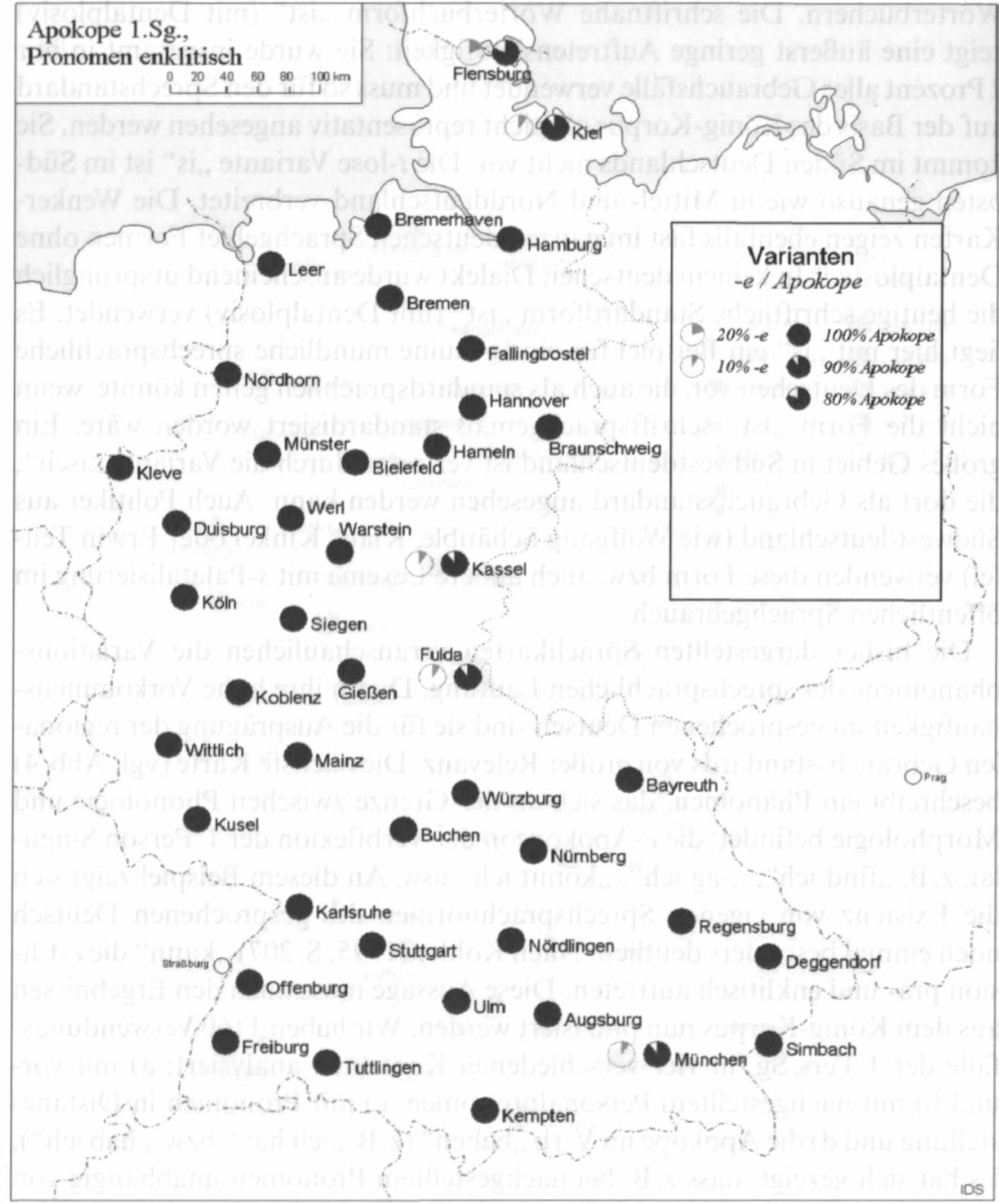

Abb. 4: e-Apokope bei nachgestelltem Pronomen (sag ich, mach ich u.a.)

Im Folgenden werden noch zwei Beispiele für typische regionale Merkmale der Gebrauchsstandards veranschaulicht. Die nächste Karte (Abb.5) beschreibt die $g$-Spirantisierung vor Plosiv wie z. B. in Wörtern trägt [tre:çt] und bewegt [bəve:çt]. In der vorgelesenen Sprache kommen diese Varianten so gut wie nicht vor, wie der Ausspracheatlas von König (1989, Bd. II, S. 305) zeigt. In der Spontansprache dagegen ist die Variante häufig belegt und es zeigen sich großräumige regionale Unterschiede zwischen Norden und Süden: Spirantisierte Varianten (nicht-normgerechte nach kodifizierten Regeln) kommen im Norden großräumig vor; nichtspirantisierte Varianten, d.h. normgerechte - nur im Süden. Am stärksten variabel im Gebrauch (bezüglich 


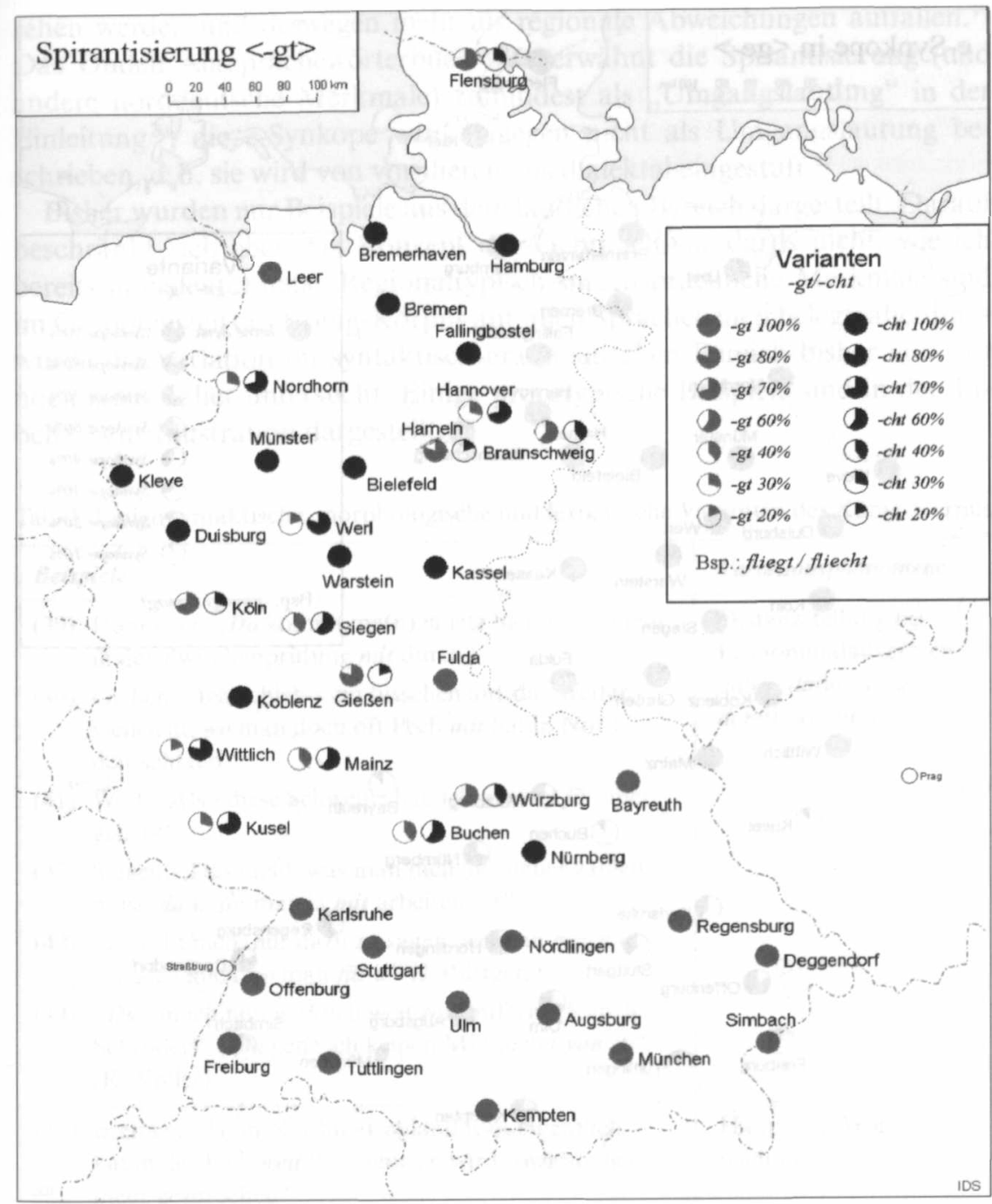

Abb. 5: Gebrauchsvarianten -gt/-cht

dieses Phänomens) ist der westmitteldeutsche Raum. Das Merkmal wird gewöhnlich als norddeutsches Schibboleth, also als typisches Kennzeichen mit hohem regionalen Symptomwert angesehen, ist aber nach den Daten des von uns untersuchten König-Korpus - wie die markierten Symbole auf der Karte 5 zeigen - auch in Mitteldeutschland und Ostfranken ein häufig belegtes Merkmal des gesprochenen Standards.

Die nächste Karte (Abb. 6) beschreibt schließlich ein weiteres großräumiges regionales Merkmal: die $e$-Synkope im schriftsprachlichen Präfix ge- (Tilgung des Vokals in der Vorsilbe ge-). Auch hier ist kein Vergleich zur Vorlesesprache (König 1989) möglich, da dieses Phänomen ein typisch spontansprachliches 


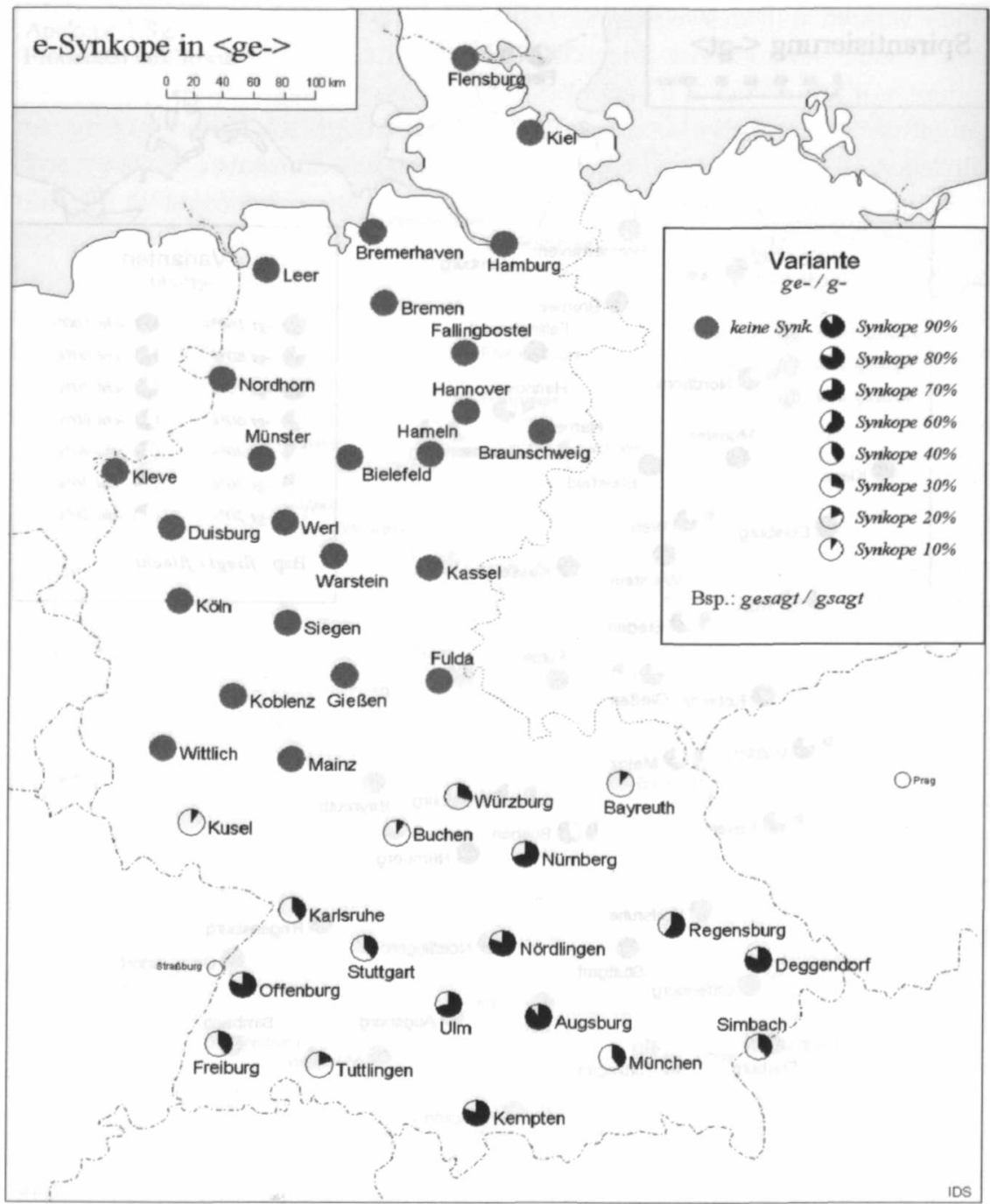

Abb. 6: ge-Synkope

ist und in der Vorlesesprache in der Regel nicht vorkommt. Die e-Synkope ist im Korpus sowohl bei Verbalpräfix belegt als auch bei Adjektiven und Substantiven. Die Karte zeigt großräumige regionale Unterschiede und eine eindeutige Nord-Süd-Zweigliederung: die Synkope wird nur im südlichen Teil des Untersuchungsgebiets vorgenommen. ${ }^{18}$ Die synkopierte süddeutsche Variante fällt im öffentlichen Sprachgebrauch mehr auf als z. B. die norddeutsche Spirantisierung. Das hängt vermutlich damit zusammen, dass süddeutsche Merkmale grundsätzlich weniger als standardsprachgemäß ange-

${ }^{18}$ Ein Beispiel aus dem König-Korpus (vgl. Tabelle2, Satz 38). 
sehen werden und deswegen mehr als regionale Abweichungen auffallen. ${ }^{19}$ Das Duden Aussprachewörterbuch z.B. erwähnt die Spirantisierung (und andere norddeutsche Merkmale) zumindest als „Umgangslautung“ in der Einleitung ${ }^{20}$; die $e$-Synkope wird dagegen nicht als Umgangslautung beschrieben, d.h. sie wird von vornherein als dialektal eingestuft. ${ }^{21}$

Bisher wurden nur Beispiele aus dem lautlichen Bereich dargestellt. Darauf beschränkt sich aber das Konzept der Gebrauchsstandards nicht, wie ich bereits angedeutet habe. Regionaltypisch-sprechsprachliche Merkmale sind im Gesprächsteil des König-Korpus auf allen Sprachebenen belegt; allerdings wurde die Variation im syntaktisch-grammatischen Bereich bisher von uns noch nicht näher untersucht. Einige prototypische Beispiele sind in der Tabelle 3 zur Illustration dargestellt.

Tab. 3: Einige syntaktische, morphologische und lexikalische Varianten des König-Korpus

\begin{tabular}{|c|c|c|}
\hline \multicolumn{2}{|c|}{ Beispiele } & \multirow{3}{*}{$\begin{array}{l}\text { Variationsphänomene } \\
\text { Distanzstellung bei } \\
\text { Pronominaladverbien } \\
\text { dabei, dafür, dazu, } \\
\text { damit, womit usw. }\end{array}$} \\
\hline (39) & $\begin{array}{l}\text { Hannover: „Da kommt ma(n) ja jetz nich ma(l) mehr } \\
\text { in der Zwischenprüfung mit durch“ }\end{array}$ & \\
\hline (40) & $\begin{array}{l}\text { Gießen: „Ich schieb-s ein bisschen auf das Wetter } \\
\text { vielleicht, wo man doch oft Pech } \text { mit hat in Nord- } \\
\text { deutschland“ }\end{array}$ & \\
\hline (41) & $\begin{array}{l}\text { Werl: „Also diese Schweine hab ich nie viel für über } \\
\text { gehabt“" }\end{array}$ & \\
\hline (42) & $\begin{array}{l}\text { Siegen: „Das Geld, was man nich als Steuer zahlen } \\
\text { muss, } \boldsymbol{d a} \text { kann man ja mit arbeiten, ne?“ }\end{array}$ & \\
\hline (43) & $\begin{array}{l}\text { „Es geht nich, nur dazu zu sagen, wo man gegen is, } \\
\text { sondern auch wo man für is" (J. Rüttgers) }\end{array}$ & \\
\hline (44) & $\begin{array}{l}\text { „Da bin ich nun wirklich weit von entfernt (Kanzler } \\
\text { Schröder)“ „Da gehe ich keinen Millimeter von ab“ } \\
\text { (R. Völler) }\end{array}$ & \\
\hline (45) & $\begin{array}{l}\text { Bremen: „Ja, in Norddeutschland is-es eigentlich } \\
\text { kaum der Fall, weil Plattdeutsch wird sowieso nich } \\
\text { mehr gesprochen“ }\end{array}$ & $\begin{array}{l}\text { Hauptsatz-Wortstellung } \\
\text { nach weil }\end{array}$ \\
\hline (46) & $\begin{array}{l}\text { Werl: „Ich hab da auch nich den absoluten Durch- } \\
\text { blick, weil ich hab mich jetzt zum dritten Mal } \\
\text { beworben und ich rück weder vor noch irgendwie } \\
\text { zurück auf der Warteliste, ne?“ }\end{array}$ & \\
\hline
\end{tabular}

19 Vgl. dazu auch die detaillierten Ausführungen von König (1996).

${ }^{20}$ Duden-Aussprachewörterbuch (2000), S. 66.

${ }^{21}$ Dazu ein Beispiel: Bayerns Ministerpräsident Stoiber verwendet gelegentlich bei Interviews im Bayerischen Rundfunk die synkopierte Variante, überregional aber weitgehend nicht, sondern dafür volles ge. Kanzler Schröder dagegen spirantisiert auch in höchsten Kontextstilen, die keineswegs als umgangssprachlich charakterisiert werden können, z. B. in Regierungserklärungen. 
Fortsetzung Tabelle 3

\begin{tabular}{|c|c|c|}
\hline \multicolumn{2}{|c|}{ Beispiele } & \multirow{2}{*}{$\begin{array}{l}\text { Variationsphänomene } \\
\text { Tempusgebrauch } \\
\text { (Doppelperfekt) }\end{array}$} \\
\hline$(47)$ & $\begin{array}{l}\text { Karlsruhe: „Von Hannover explizit noch nich, nein, } \\
\text { hab ich noch nie so ghört ghabt" }\end{array}$ & \\
\hline (48) & $\begin{array}{l}\text { Augsburg: „Was haben sie denn da so gelernt, das tät } \\
\text { mich mal interessieren“" [nt.] }\end{array}$ & tun-Konjunktiv \\
\hline (49) & $\begin{array}{l}\text { Werl: „Nein, ich fand das sogar, als ich hier nach Frei- } \\
\text { burch } \text { kam, fand ich das unheimliches Hochdeutsch, } \\
\text { was ich da sprach." }\end{array}$ & $\begin{array}{l}\text { Formenpräferenz } \\
\text { Präteritum }\end{array}$ \\
\hline$(50)$ & $\begin{array}{l}\text { Karlsruhe: „Weil also verschiedene Bundesländer } \\
\text { doch einige Anforderungen mehr stellen in Pädagogik } \\
\text { wie z.B. Baden-Württemberg“ }\end{array}$ & $\begin{array}{l}\text { Komparativpartikel } \\
\text { wie-als }\end{array}$ \\
\hline$(51)$ & Ulm: „Dann hat sich des quasi a bissle erhalte“ & (-chen,-lein) \\
\hline$(52)$ & Nördlingen: ,Nee!"“[...],,Naa, naa!““ & $<$ nein $>$ \\
\hline (53) & $\begin{array}{l}\text { Kusel: „Ach nee, (ich) weiß net, s is halt so.“- } \\
\text { „Nein, danke!“ }\end{array}$ & nein-nee - naa-'a'a \\
\hline (54) & $\begin{array}{l}\text { Werl: „Und dann ham-wer-s uns überleecht, ob-wer } \\
\text { doch nich hierhin könnten“ }\end{array}$ & $\begin{array}{l}\text { hierhin-hierher } \\
\text { Lexempräferenz }\end{array}$ \\
\hline
\end{tabular}

Das sind in der Syntax Wortstellungsregularitäten wie die Distanzstellung bei Pronominaladverbien davon, damit, dagegen, dafür usw. (Beispielsätze 39-44). Diese syntaktische Variante breitet sich gegenwärtig aus, wird insgesamt aber als typisch norddeutsch betrachtet (Stellmacher 2001, S. 28). Sie ist auch für Politiker im öffentlichen Sprachgebrauch typisch (Beispiele 43-44). In der Morphologie sind das besondere Formen des Plusquamperfekts (die Variante Doppelperfekt im Beispiel47). Die Konjunktiv-Variante mit dem Verb tun ist durch ein Beispiel aus Augsburg dargestellt (Satz 48). Regionale Unterschiede bestehen auch bei Gebrauchspräferenzen, z. B. von Präteritum im Norden gegenüber einer vermehrten Verwendung des Perfekts im Süden (Beispiel 49). Trotz des eingeschränkten Umfangs des untersuchten Test-Korpus lassen sich auch einige lexikalische Unterschiede feststellen, die eine signifikante regionale Verteilung des Wortschatzes in den Sprachgebrauchsstandards bestätigen.

\section{Wie viele regionale Gebrauchsstandards müsste/könnte man unterscheiden und lehren?}

$\mathrm{Zu}$ Beginn des dritten Abschnitts möchte ich noch einmal kurz auf die Position von Durrell (2003 u.a.) in Bezug auf das Englische verweisen. Er stellt für das Englische einen sprechsprachlichen Gebrauchsstandard fest, der sich durch bestimmte Sprachmerkmale auszeichnet und der realistischerweise 
auch im Fremdsprachenunterricht gelehrt werden muss. Etwas Analoges fordert er für das Deutsche, und zwar nicht zuletzt deshalb, um Deutschlernern ein realistisches Bild von der Sprachgebrauchssituation in Deutschland zu vermitteln. Ich schließe mich der Einschätzung Durrells grundsätzlich an. Wir brauchen auch für das Deutsche eine Beschreibung des sprechsprachlichen schriftfernen Standards. Allerdings ist dieser sprechsprachliche Standard regional geprägt - was jedoch nicht gleichzusetzen ist mit dialektal, wie die oben erläuterten Beispiele verdeutlicht haben. Ich widme mich im Folgenden der Frage, wie viele regionale Gebrauchsstandards man im gegenwärtigen Deutschen denn differenzieren müsste bzw. könnte. ${ }^{22}$

Es gibt nach unserem Material sicherlich einen großräumigen norddeutschen Gebrauchsstandard. ${ }^{23}$ Er ist relativ einheitlich und auch relativ nah zum Schriftdeutschen und weist insgesamt keine große Variationsvielfalt auf. Im Vergleich zum Wortatlas der deutschen Umgangssprachen von Eichhoff scheint die südliche Grenze dieses Raumes teilweise nach Süden vorgerückt zu sein. Berücksichtigt man die Expansion der norddeutschen Varianten in das Mittel- und Südwestdeutsche, seine relative Nähe zum Schriftdeutschen und die vergleichsweise geringe, aber konsequent ausgeprägte Variation, dann ist nach unserem jetzigen Kenntnisstand in diesem Raum ein Sprechstandard mit einer norddeutschen Gebrauchsnorm („Nordnorm“) feststellbar.

Anders als im Norden, stellt sich das Oberdeutsche beim regionalen Gebrauchsstandard nicht als einheitlicher Raum dar. Von einer "Südnorm“ kann nur bedingt gesprochen werden, denn es ist im Süden eine Ost/WestDifferenzierung zu erkennen. Es lassen sich hier zwei Sprechstandards feststellen.

a) Im Südosten, ungefähr auf dem Gebiet des heutigen Bundeslandes Bayern, zeichnet sich nach den Daten bezüglich der untersuchten Merkmale ein ziemlich einheitlicher bayerischer Gebrauchsstandard ab. Franken und Bayerische Schwaben sind bekanntlich keine sprachlichen, sondern politische Bayern - weil sie nach administrativer Einteilung zum Land Bayern gehören, jedoch eine von Altbayern deutlich abweichende dialektale Basis aufweisen. Dieser sprachliche Unterschied lässt sich im Standardsprachgebrauch nach vorliegenden Daten nicht in dieser Konsequenz beobachten. Es entsteht der Eindruck, dass die Sprecher aus diesen Regionen einen gemeinsamen Sprachkonservatismus zeigen, der sich vielleicht auch aus der gemeinsamen Identität als „Staatsbayern“ ergibt. ${ }^{24}$ Unter Sprachkonservatismus verstehen wir hier die Tatsache, dass der Raum vergleichsweise ver-

22 Der Vorschlag zur regionalen Differenzierung des bundesdeutschen Raumes basiert auf den bisher vorliegenden Untersuchungsergebnissen (Analysen und Karten) des KönigKorpus. Stand: März 2004.

23 Vgl. dazu auch Stellmacher (1997) und Stellmacher (2001).

${ }^{24}$ Zum Einfluss des Bairischen auf die Sprache Bayerisch-Schwabens vgl. jetzt Kleiner (2003). 
schlossen ist gegenüber sprachlichen Neuerungen aus dem Norden. Der bayerische Gebrauchsstandard ist auch aus diesem Grunde schriftferner als in Norddeutschland.

b) Obwohl das Schwäbisch-Alemannische im südwestlichen Teil nach den Daten des König-Korpus teilweise instabil erscheint, nehmen wir auch hier einen eigenen Gebrauchsstandard an, der sich ungefähr mit dem Gebiet des Bundeslandes Baden-Württemberg deckt. (Dies ist im übrigen auch der Sprachraum, für den der Werbeslogan Wir können alles - außer Hochdeutsch das Vorhandensein eines regional geprägten Standards geradezu als Markenzeichen in Anspruch nimmt.) Die Besonderheit dieses Raumes äußert sich in einer spezifischen Kombination einerseits von eigenen und andererseits von außen übernommenen sprachlichen Merkmalen. Darin besteht die Eigenständigkeit oder auch die Dynamik (bzw. Instabilität) der südwestdeutschen Norm, wodurch die Sprachregion variantenreicher erscheint als z.B. der Südosten. Als Gründe für die „Aufnahmefreudigkeit“ des Südwestens für standardnähere (und damit in der Regel norddeutsche) Formen ist möglicherweise ein Sprachbewusstsein zu nennen, das schon viel früher als in Bayern eine Tendenz zur Aufgabe regionalsprachlicher Merkmale aufgewiesen hat. Das hat offensichtlich zur Folge, dass der Gebrauchsstandard in Südwestdeutschland eine merkliche Annäherung an das Standarddeutsche erfahren hat, ganz im Gegensatz zu Bayern, wo man mit dem Slogan Mir san mir die regionale Identität häufig auch mit entsprechenden sprachlichen Mitteln zu untermauern pflegt.

Schwieriger ist es, einen eigenständigen mitteldeutschen Gebrauchsstandard anzunehmen. Immer wieder begegnet in den Kartenbildern ein Nord/SüdKontrast, der aber im Gegensatz zur Lautverschiebungslinie, die das Niederdeutsche vom Mitteldeutschen trennt, viel weiter südlich anzusetzen ist. Dieser Kontrast lässt sich an einem Linienbündel feststellen, das sich nördlich und südlich der Mainlinie staffelt und maximal zwischen Fulda und Gießen im Norden und Nürnberg und Karlsruhe im Süden pendelt. Die Besonderheit des mitteldeutschen Raumes besteht nach den vorliegenden Daten vor allem darin, dass der Sprachgebrauch sich je nach Merkmal entweder nach der Nordnorm oder nach der Südnorm richtet..$^{25}$ Große Teile des traditionellen Mitteldeutschen, z. B. Mainz, Koblenz, Köln gehen mit dem Norden, d.h. ihr regionaler Gebrauchsstandard unterscheidet sich in den bisher von uns untersuchten Merkmalen nicht von dem des Nordens. Nach Süden reicht dieser Interferenzraum besonders im Ostfränkischen in das Oberdeutsche hinein (Buchen, Würzburg, Nürnberg, Bayreuth). In dem von uns untersuchten Material zeigt sich zwar eine deutliche Tendenz zum Norden, aber als norddeutscher Standard im oben beschriebenen Sinn kann der traditionelle mitteldeutsche Raum jedoch nach dem jetzigen Kenntnisstand nicht klassifi-

${ }^{25} \mathrm{Zu}$ ähnlichen Ergebnissen kommt auch Dingeldein (2001), S. 47. 
ziert werden. Der mitteldeutsche Raum bedarf also besonders einer weiteren Untersuchung und Beschreibung, um Aufschlüsse über seinen Status als eigenständiger Gebrauchsstandard zu erhalten. Vorläufig kann man angesichts der Datenlage zumindest davon ausgehen, dass im mitteldeutschen Raum - gerade weil er als breites Interferenzgebiet zwischen nördlichen und südlichen Formen fungiert - ein eigenständiger, regional differenzierter $\mathrm{Ge}$ brauchsstandard existiert.

Auf der Grundlage der Ausführungen würde ich somit vorläufig vier regionale Gebrauchsstandards für das Deutsche (in der alten Bundesrepublik Deutschland) differenzieren, wie sie auf der Karte (Abb.7) zu sehen ist: 1) einen sicheren, relativ schriftfernen Gebrauchsstandard in der Nordhälfte Deutschlands; 2) einen stabilen, schriftfernen Gebrauchsstandard im Südosten Deutschlands; 3) einen labilen, neuerungsfreudigen Gebrauchsstandard im Südwesten Deutschlands; 4) einen regional differenzierten, wenig eigenständigen Gebrauchsstandard in einem Interferenzraum in der südlichen Mitte Deutschlands. 


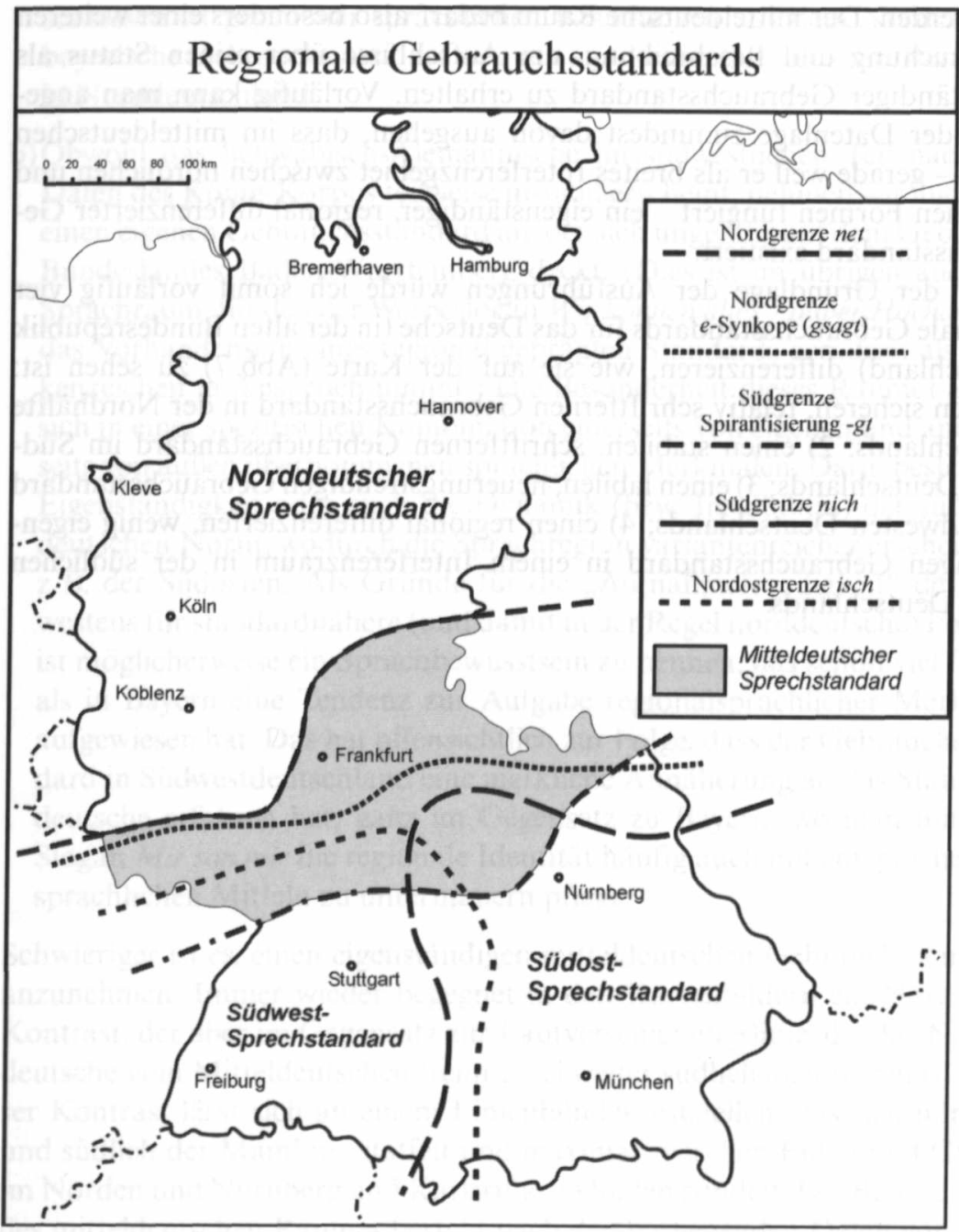

Abb. 7: Regionale Gebrauchsstandards

Anmerkung zur Karte: Diese Karte basiert auf den spontansprachlichen Daten des König-Korpus und stellt eine grobe Einteilung der regionalen Gebrauchsstandards des Deutschen dar. Diese Einteilung ergibt sich durch die Isoglossen einiger großräumiger Varianten, die im Text ausführlich erläutert wurden, und weiterer sprechsprachlicher Phänomene, die in Tabelle 1-3 zusammengefasst sind. Nicht berücksichtigt sind die von König (1989) beschriebenen Merkmale der Aussprache des Schriftdeutschen, weil diese Merkmale allein nicht zur arealen Definition regionaler Gebrauchsstandards ausreichen. Deren Isoglossen decken sich jedoch in vielen Fällen mit den hier dargestellten. Für ein differenzierteres Bild sind weitere Analysen notwendig. 


\section{Forschungs- und Beschreibungsaufgaben}

Angeregt durch Sprachbeschreibungen, die für das britische Englisch existieren, ${ }^{26}$ muss auch für das Deutsche eine Sprachschicht konzipiert und beschrieben werden, die relativ schriftsprachfern ist und dennoch die Dialekte und dialektgeprägten Umgangssprachen überlagert. Diese Sprachschicht kann als schriftferner, informeller, sprechsprachlicher Standard charakterisiert werden. Das entspräche dem Konzept des „colloquial english “, also: „colloquial german“. Ich schlage den Ausdruck „deutscher Sprechstandard“ vor. Im Unterschied zum Englischen kann man allerdings bezüglich des Deutschen nicht von einem völlig überregionalen „colloquial german“ sprechen. Man muss vielmehr großräumige regionale Unterschiede annehmen, wie die oben angeführte Beispiele auf den Sprachkarten zeigen. Es handelt sich also um „regionale Gebrauchsstandards“.

Es gibt verschiedene Gründe, die großräumigen regionalen Gebrauchsstandards zu erforschen und zu beschreiben. Zwei dieser Gründe sind:

a) Die herkömmlichen und bis heute tradierten Konzepte über die Sprachverhältnisse im Deutschen entsprechen einfach nicht mehr den Sprachgebrauchsrealitäten, d.h. es gibt Unzulänglichkeiten in der Beschreibung des heutigen Deutsch. Im traditionellen Betrachtungsschema der Variation (vgl. Abschnitt 1) wird auf oberster Ebene ein schriftsprachlich orientierter Standard, gewissermaßen eine Hochsprache, angenommen. Dann - auf der zweiten Ebene - folgen regionale Umgangssprachen. Mihm (2000) skizziert aufgrund der Forschungslage in der Umgangs- und Regionalspracheforschung allein fast fünfzig verschiedenartige groß- und kleinräumige Umgangssprachen. Dieses Bild ergibt sich aus regionalen Einzelforschungen, die bekanntlich sowohl empirisch als auch theoretisch sehr unterschiedlich ausgerichtet waren.

b) Neben den theoretisch-wissenschaftlichen Defiziten muss vor allem auf die Sprachlehrsituation hingewiesen werden: Es ist realitätsfern und deshalb ineffektiv, wenn Deutschlernende mit Standards bzw. Normen konfrontiert werden, die nicht den sprachlichen Realitäten in Deutschland entsprechen, sondern lediglich eine idealisierte Norm wiedergeben. Der deutsche Sprechstandard ist die Sprachform, die u.E. in Zukunft im Bereich „Deutsch als Fremdsprache" gelehrt werden muss. Es ist dann auch die Sprachform, die in den gängigen Wörterbüchern des Deutschen zunehmend deutlich beschrieben werden sollte. Dies wird auch von v. Polenz (1998) hervorgehoben, wenn er schreibt:

„Da Standardsprache heute nicht mehr nur am Schreibstil und der Sprechstandard nicht mehr am Vorbild von Pastoren, Festrednern, Schauspielern und traditionellen Nach-

${ }^{26}$ Durrell (2003) führt das Beispiel eines 2000 erschienenen englischen Lehrbuchs für Anfänger an, in dem ,der Lerner mit typischen Merkmalen der alltäglichen britischen Sprechsprache in völlig natürlichen Gesprächen konfrontiert wird“ (Hinweis bei Durrell 2003, S. 250, Fußnote 26). 
richtensprechern orientiert ist, muss das Vorkommen umgangssprachlicher Merkmale in öffentlicher Rede als teilweise Annäherung der Standardsprache an die Umgangssprache, zumindest als Entstehung eines sozial nicht mehr diskriminierten Substandards erklärt werden. Dieser Substandard steht textsorten- und situationsspezifisch Allen wahlweise zur Verfügung, erscheint oft funktional angemessener und sollte deshalb auch in Deutsch als Fremdsprache gelehrt werden" (S. 358).

Gegenwärtig arbeiten wir an der Sammlung und systematischen Bearbeitung von Sprachdaten zu den regionalen Gebrauchsstandards. ${ }^{27}$ Unser Ziel ist es, ganz praktische Vorschläge zu machen, wie man in die marktgängigen (etablierten) Wörterbücher und auch Unterrichtsmaterialien Informationen einfügt über den tatsächlichen deutschen Sprechstandard. Als Vorbild könnten manche Lehr- und Wörterbücher der englischen Sprache dienen, die ihr Ziel darin sehen, nicht nur eine starre formelle Standardsprache darzustellen und zu lehren, wie z. B. das Longman Pronunciation Dictionary (LPD, s. Wells 2000). Vergleicht man einmal die Darstellungsziele des LPD mit denen des Duden-Aussprachewörterbuchs, so wird der grundlegende Unterschied zwischen beiden Herangehensweisen deutlich: Während das Duden-Wörterbuch im Vorwort ankündigt, dass die Varianten ausgeblendet werden oder auf ein Mindestma $\beta$ beschränkt, sieht das LPD geradezu seine Aufgabe darin, Varianten (,alternative pronunciations“) anzugeben, die im modernen Sprachgebrauch des Englischen vorkommen. Unser größeres Ziel ist, ein Textkorpus im IDS aufzubauen, das eine weitere empirische Grundlage für die Beschreibung von regionalen Sprechstandards bieten kann. Wir denken an punktuelle Aufnahmen von Sprechereignissen, die die Charakteristik und Verteilung von sprechsprachlichen Standards im deutschländischen Deutsch zeigen. Dabei gehen wir davon aus, dass das deutschländische Deutsch nur dann adäquat und angemessen beschrieben werden kann, wenn die regionalen Standardvarietäten, die es konstituieren, berücksichtigt werden. So betrachtet auch von Polenz (1996, S. 217) die Erforschung von regionalen Gebrauchsstandards, wie wir sie vorschlagen, als umfassendes Forschungsdesiderat und Fernziel: „Wenn hierbei Regionalismen einbezogen werden, müssen neben norddeutschen gleichermaßen auch süddeutsche, rheinische, hessische, sächsische usw. berücksichtigt werden“.

\section{Literatur}

Ammon, Ulrich (1995): Die deutsche Sprache in Deutschland, Österreich und der Schweiz. Das Problem der nationalen Varietäten. Berlin.

Auer, Peter (1997): Führt Dialektabbau zur Stärkung oder Schwächung der Standardvarietät? In: Mattheier, K. J./E. Radtke (Hrsg.): Standardisierung und Destandardisierung europäischer Nationalsprachen. Frankfurt/Main. S. 129-161.

27 Siehe dazu auch Berend (2003). 
Barbour, Stephen/Patrick Stevenson (1998): Variation im Deutschen. Soziolinguistische Perspektiven. Berlin/New York.

Bellmann, Günter (1983): Probleme des Substandards im Deutschen. In: Mattheier, Klaus J. (Hrsg.): Aspekte der Dialekttheorie. Tübingen. S. 105-130.

Berend, Nina (2003): Aussprachevarianten des Deutschen. Überlegungen zur Gestaltung einer korpusbasierten Datenbank. In: Androutsopoulos, Jannis K./Evelyn Ziegler (Hrsg.): „Standardfragen“. Soziolinguistische Perspektiven auf Sprachgeschichte, Sprachkontakt und Sprachvariation. Frankfurt/Main. S. 235-249.

Dingeldein, Heinrich J. (1997): Sprachvarietäten in „Mitteldeutschland“. Gebrauch und Räumlichkeiten. In: Stickel, Gerhard (Hrsg.): Varietäten des Deutschen. Regionalund Umgangssprachen. Berlin/New York. S. 109-141.

Dingeldein, Heinrich (2001): Zum heute gesprochenen Deutsch im mittleren Deutschland. In: Knipf-Komlosi, E./N. Berend (Hrsg.): Regionale Standards. Budapest. S. 41-60. DiWA (= Digitaler Wenker-Atlas), Marburg. http://www.diwa.info/

Duden-Aussprachewörterbuch. 4. Aufl. Bd. 6, 2000.

Durrell, Martin (2003): Using German. A guide to contemporary usage. 2. Aufl. Cambridge.

Durrell, Martin (1995): Sprachliche Variation als Kommunikationsbarriere. In: Popp, Heidrun (Hrsg.): Deutsch als Fremdsprache. An den Quellen eines Faches. Festschrift für Gerhard Helbig zum 65. Geburtstag. München. S. 417-428.

Durrell, Martin (1999): Standardsprache in England und Deutschland. In: ZGL 27, 5, S. 261-284.

Durrell, Martin (2003): Register, Variation und Fremdsprachenvermittlung. In: Stickel, Gerhard (Hrsg.): Deutsch von außen. Berlin. S. 239-258.

Eichinger, Ludwig M. (2001): Sprache und Sprachgebrauch im Süden Deutschlands. Konturen eines süddeutschen Gebrauchsstandards. In: Knipf-Komlosi, E./N. Berend (Hrsg.): Regionale Standards. Budapest. S.61-94.

Eichhoff, Jürgen (1977/2000): Wortatlas der deutschen Umgangssprachen. Band 1-4. Bern/ München.

Eisenberg, Peter (1998): Grundriss der deutschen Grammatik. Bd. 1: Das Wort. Stuttgart.

Götz, Ursula (1995): Regionale grammatische Varianten des Standarddeutschen. In: Sprachwissenschaft 20, S. 222-238.

Kleiner, Stefan (2003): Bairisches in der Regionalsprache Bayerisch-Schwabens: Die Übernahme des Flexionssuffixes (-ts) für die 2. Person Plural. In: Funk, Edith/Stefan Kleiner/Manfred Renn/Bernadette Wecker (Hrsg.): Sprachgeschichten. Ein Lesebuch für Werner König zum 60. Geburtstag. Heidelberg. S. 137-151.

Kohler, Klaus J. (1995): Einführung in die Phonetik des Deutschen. 2., neu bearbeitete Auflage. Berlin. (= Grundlagen der Germanistik 20).

König, Werner (1989): Atlas zur Aussprache des Schriftdeutschen in der Bundesrepublik Deutschland. Ismaning.

König, Werner (1996): dtv-Atlas zur deutschen Sprache. München.

König, Werner (1997): Phonetisch-phonologische Regionalismen in der deutschen Standardsprache. Konsequenzen für den Unterricht, Deutsch als Fremdsprache'? In: Stickel, Gerhard (Hrsg.): Varietäten des Deutschen. Regional- und Umgangssprachen. Berlin/New York. S. 246-270.

Mattheier, Klaus/Edgar Radtke (Hrsg.) (1997): Standardisierung und Destandardisierung europäischer Nationalsprachen. Frankfurt/Main.

Mihm, Arend (2000): Die Rolle der Umgangssprachen seit der Mitte des 20. Jahrhunderts. In: Besch, Werner/Betten, Anne/Reichmann, Oskar/Sonderegger, S. (Hrsg.): Sprachgeschichte. 2. Aufl. Berlin/New York. S. 2107-2137. 
Polenz, Peter v. (1996): Österreichisches, schweizerisches, deutschländisches und teutonisches Deutsch. In: ZGL 24, S. 205-220.

Polenz, Peter v. (1999): Deutsche Sprachgeschichte vom Spätmittelalter bis zur Gegenwart. Band III. 19. und 20. Jahrhundert. Berlin.

Renn, Manfred (1999): Das Allgäuer Dialektbuch. Augsburg.

Stellmacher, Dieter (1997): Sprachsituation in Norddeutschland. In: Stickel, Gerhard (Hrsg.): Varietäten des Deutschen. Regional- und Umgangssprachen. Berlin/New York. S. 18-40.

Stellmacher, Dieter (2001): Die deutsche Sprache in Norddeutschland. In: Knipf-Komlosi, E./N. Berend (Hrsg.): Regionale Standards. Budapest. S. 18-40.

Wells, J.C. (2000): Longman Pronunciation Dictionary. Harlow.

Wiesinger, Peter (Hrsg.) (1988): Das österreichische Deutsch. (= Schriften zur deutschen Sprache in Österreich, Bd. 12). Wien.

Wolf, Norbert Richard (1994): Österreichisches zum österreichischen Deutsch. Aus Anlass des Erscheinens von Wolfgang Pollak: Was halten die Österreicher von ihrem Deutsch? In: ZDL 61, S. 66-76. 